\title{
Abnormal Crowd behavior Detection Using Structural Context Descriptor
}

\author{
Pooja and Sarvesh Vishwakarma
}

\begin{abstract}
In concern with the people safety in public places, an Abnormal Behavior Detection in crowded scene has gained popularity. The Abnormal Behavior Detection is an active area of research. This has gained a particular interest amongst the researchers in this evolving era of computer science. For this purpose the system proposes the structure context descriptor (SCD) for describing the crowd individual, which represents the relationship among the individuals. The approach presents the abnormal behavior detection method from the crowd scenes to efficiently detect anomalies present in the crowded scenes.
\end{abstract}

Keywords--- Crowded Scenes, Abnormal Behavior Detection, Tracking, computer Vision.

\section{INTRODUCTION}

$\mathrm{T}$ WE crowded scenes are more common as the human population escalates reliably. Nowadays everywhere we can find the crowded scenes in this universe. It has attracted the importance of the researchers from last few decades. The safety, security and management of the peoples in the public places like airport, railway station and shopping malls have been a big challenge. The abnormality detection in the crowded scenes benefits significance [1].

The introduction of the vigilance cameras becomes a common task which automatically detects the abnormal crowd behavior, thus providing the security. The vigilance work is to invigilate the movement of every person in that event. Yet, it is slight difficult for the crowd events of high density. The occlusion [2] is one of the severe obstacles, where detecting and tracking of the individual becomes tedious and cumbersome. Another problem is crowd behavior definition. Since we can encounter lot of crowd behaviors, however defining each of them is impossible.

Humans are capable in extracting information of behavior patterns in vigilance area, real time monitoring of scenes for the irregular contexts and gives potential for immediate response [15].

Tracking a person or an object is always very tedious and sometimes unfeasible in the case of high-density crowded scenes [16]. However, they experience an enormous drawback in their ability of monitoring or observing the simultaneous signals. The abnormality is very laboriousto construe. Hence

Pooja, Student, Department of CSE, SCEM, Mangaluru. E-mail:pooji.k.acharya34@gmail.com

Sarvesh Vishwakarma, Professor, Department of CSE, SCEM, Mangaluru.E-mail:sarvesh.vish@gmail.com

DOI: 10.9756/BIJAIP.10466 it is very tedious task to define a general method for crowd abnormality detection.

The Researchers has enthralled their attention towards the abnormality detection, a key facet of crowd scene analysis. Though the problem is very vast but the efforts in research are dispersed in approaches, assumptions, objectives and interpretation of a particular problem.

\section{A. Problem Statement}

Detection of abnormal behavior in crowd scenes is challenging and laborious. An Abnormal behavior in crowded scenes interprets a helpful explanatory evidence for the different applications, such as intelligent surveillance, safety evaluation, behavior analysis etc. In this proposed approach, Structural Context Descriptor (SCD) is used to depict every person in the crowd.

\section{B. Motivation}

Detecting an abnormal behavior in crowded scenes is an active area of research in the field of computer vision. The specific idea of abnormality is tedious to interpret as various peculiar operations convoluted. Hence, it is still very challenging to model an ordinary method for abnormality in crowd behavior detection.

The very important concentration here is on the abnormal detection in the crowd behavior. In consideration of various approaches towards this direction, some extract sudden motion of individuals to localize the abnormality and others promotes object paths which hardly appear to be abnormal. The first group utilizes the Optical Flow (OF) to extract image motion clues. The second group needs efficient tracker to obtain paths. Because of the high density of crowd scenes trajectory based methods are difficult to implement. The crowd is defined as "group of people who are related to one another", the work probes crowd anomaly detection from crowd structure modelling. For crowd structure extraction, SCD is proposed to exploit context clues between individuals.

\section{LITERATURE SURVEY}

\section{A. Introduction}

The regular hike in the human folk leads to the crowded scenes. All over the universe crowd scenarios could be noticed. This has gained the attraction of the researchers from few decades. The protection, care and control of the people in public place like heliport, rail line, plaza has become quite serious and challenging task to handle the overcrowding. Thus the deviation of the people from the normal behavior which in turn called as abnormal, in the crowded scenes aids some significance [3]. 
The care must be taken by introducing the vigilance cameras which discovers the abnormal behavior in crowd undoubtedly [4]. The Researchers are completely focused towards this abnormal detection, an important feature of the crowd scene analysis. The problem is very big, but the work in research highlightsthe issue in various ways, beliefs, goals and understanding of difficulties.

\section{B. Crowd Anomaly Detection}

Crowd anomaly detection is recognized at two levels:data with labels; unlabeled data. The anomaly detection categorized into two classes like global anomaly detection and local anomaly detection [2], based on the presence or absence of an Abnormal Behavior. The Table 1 gives the brief review of Abnormal Detection Approaches.

\section{a. Global Anomaly Detection}

The abnormal events such as explosions, disasters, fires where people try to escape, which might cause the crowd to be dispersed totally in different positions and states. The distinguistion of abnormal state from normal is carried on by global anomaly detection. It becomes significant for global anomaly detection system to detect the presence of abnormal behavior in scene and also to determine the stop and play of events and also the transition amongst them. The Chen et al. [8], all unique area is aforethought as vertex and the human crowd is depicted graphically.

\section{b. Local Anomaly Detection}

Local anomaly detection proposes the method to know where the anomaly happens. The Method is divided into two classes:

\section{The Vision Based Approach}

The visual features are extracted and set of clusters built to represent several patterns of events.

Hidden Markov Model (HMM): The HMM considers the congenitally dynamic essence of recognized characteristics. HMM is appropriate in video event detection plus anomaly detection. Kratz and Nishino [10] has proposed a schema for designing local Spatio-Temporal movement behaviors in highly crowded scenes [12].

Dynamic texture model: It is a Spatio-Temporal generative design for video. It appears as a video series as a consideration from a linear dynamical entity, and display Spatio-Temporal [20] stationary attributes. The survey carried out till now, shows that dynamic texture is very much suited for local irregular activity detection in crowded scenes [6] Optical Flow.

Bag-of words: The typical method in abnormal detection is to employ local Spatio-Temporal video capacity based Bag of Words (BoWs) approach. This technique generally clips local low level characteristics like movement and texture. Either by developing a pixel-level background design and behavior plans or by applying spatio temporal video capacity [14].
Sparse Representation Model: In recent times, Cong et al. [13] proposed a new principle for unusual event detection depending on the Sparse Reconstruction Cost (SRC) for Multilevel Histogram of Optical Flow.

Manifold Learning Model: The manifold learning based structure are been enforced for unusual event detection in crowded scene. To learn the local movement format of the event, a Spatio-Temporal Laplacian Eiganmap approach is on board. In addition, a pair wise graph is designed by taking into account the visual context of many local patches spatial and temporal domain [17].

\section{The Physics Inspired Approach}

The several models have been proposed for representation of crowds and they are also used and connected with machine learning methods for abnormality detection.

Flow Field Model: Generally, we need to know how crowds change with respect to times and try to discover some common designs. In this manner, we could anticipate crowd abnormal behaviors [18].

$\mathrm{Wu}$ et al. [9] presented an approach for crowd flow design and the abnormal detection for structured and unstructured scenes. The work flow starts with particle advection build on optical flow and the particle paths are grouped to gain representative paths for crowd flow.

Social Force Model: For the examination and imitation of crowd SFM is on board selected for the research. Mehran et al.[5], brought up a typical approach of detecting and localizing the unusual behavior in crowd video by utilizing SFM. For this intention, Ali et al. [11], proposed an approach that is used to calculate particle flows, their interaction forces are computed using SFM. This interaction force is later generalized into image plane to get force flow for each pixel in each frame [7].

Crowd energy model: Crowd has its own features. Example, local density and velocity are important criteria for calculating crowd dynamics. Cross Histogram is used to give characteristic vector instead of parallel merging the magnitude histogram and direction histogram [19]. Then SVM (Support Vector Machine) and median filter are adopted to detect anomaly.

Table 1: Brief Review of Abnormal Detection Approaches

\begin{tabular}{|l|l|l|l|l|}
\hline S.No & Author & Year & Method & Usage \\
\hline 1 & Wang et al. & 2012 & HSFT and HMM & Local \\
\hline 2 & Li et al. & 2013 & MDT & Local \\
\hline 3 & Cong et al. & 2012 & SRC & Global \\
\hline 4 & Chen et al. & 2013 & $\begin{array}{l}\text { Eigan value of } \\
\text { graph analysis }\end{array}$ & Global \\
\hline 5 & Mehran et al. & 2009 & SFM & Local \\
\hline 6 & Wu et al. & 2010 & Choatic Invariants & Local \\
\hline 7 & Yang et al. & 2012 & $\begin{array}{l}\text { Local Pressure } \\
\text { Model }\end{array}$ & Global \\
\hline
\end{tabular}




\section{METHODOLOGY}

The flow diagram of the crowd abnormal behavior detection method is been designed. The figure 1 illustrates the steps with the detailed explanation as follows:

- Pedestrian Detection: For the given input video, frames are produced and the target is obtained. These targets are highlighted. The obtained targets are assigned a common scale to overcome the impact of the template scale for organization of the expected targets.

- Structural context Descriptor: The Structural context Descriptor (SCD) is defined to depict each person in the crowd. To do this it brings the concept of Potential Energy Function of Particle's Inter force (PEF-PIF) in solid-state physics in order to model the connection amidst the obtained target and the other people.

- Anomaly detection: The numerical value of the target might change in each frame with respect to time, which results in change in the dimension of the SCD for the next frame. The abnormality is depicted by the spatial-temporal analysis of the SCD variation.

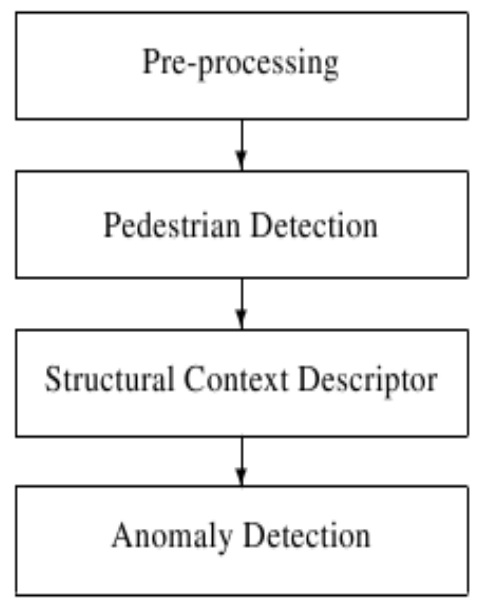

Figure 1: Typical Flow of the Proposed Method

\section{RESULTS}

The input video consists of video frames. The frames gives us the feel of the objects appearing very fast in the blink of the eyes. Thus frames are the major object existing in the video file. The basic step of conversion of video into frames and then store the converted frames in the folder through the matlab.

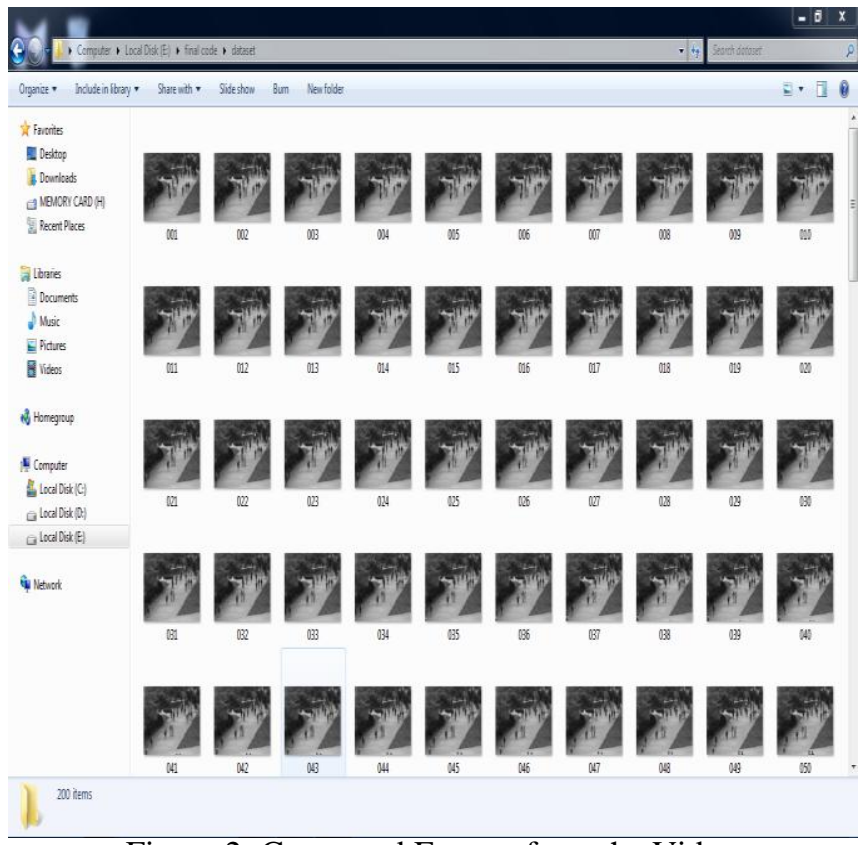

Figure 2: Converted Frames from the Video

The dataset considered here is the UCSD and the UMN datasets. The image frame is given as the input. The image segmentation is performed. The original input image is being shown in the figure 3 .

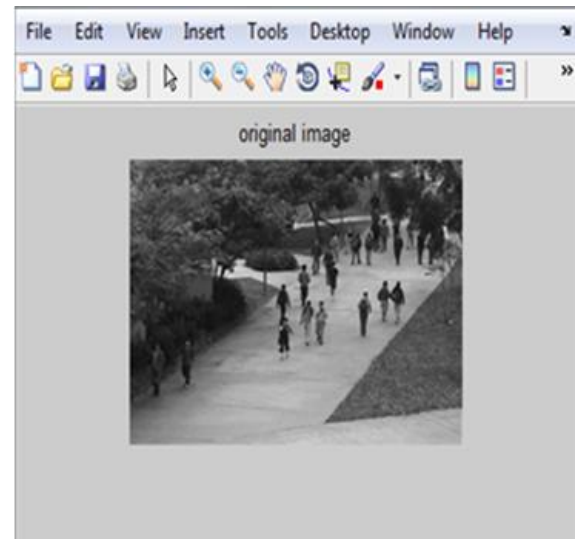

Figure 3: Output of the Original Image

The output result of the segmented image is being shown in the figure 4 .

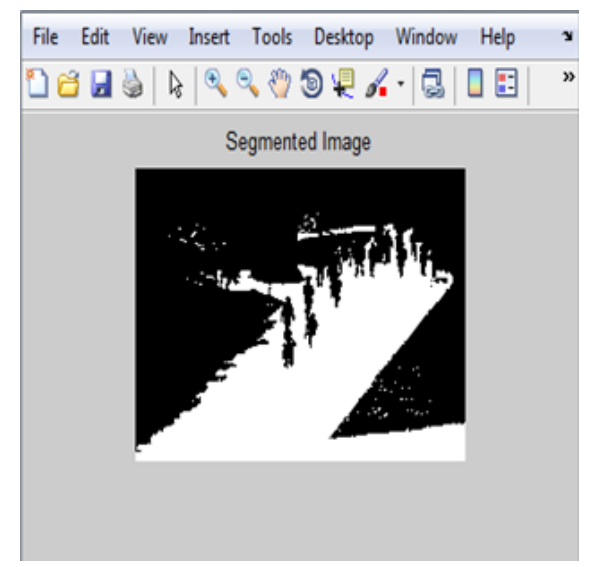

Figure 4: Output of the Segmented Image 
The background subtraction is done from the original video in order to separate the objects of foreground for the specific scene. The foreground objects are nothing but the moving objects in the scene. Recognizing the moving object in the given video sequence is a basic and also the crucial job in the field of computer vision. One common approach is that remove the background from the video so that only moving object is highlighted from the part of the video frames, thus isolating the foreground from the background. The figure 5 shows the frames of the original video along with the background separated from it. The UMN dataset is considered.

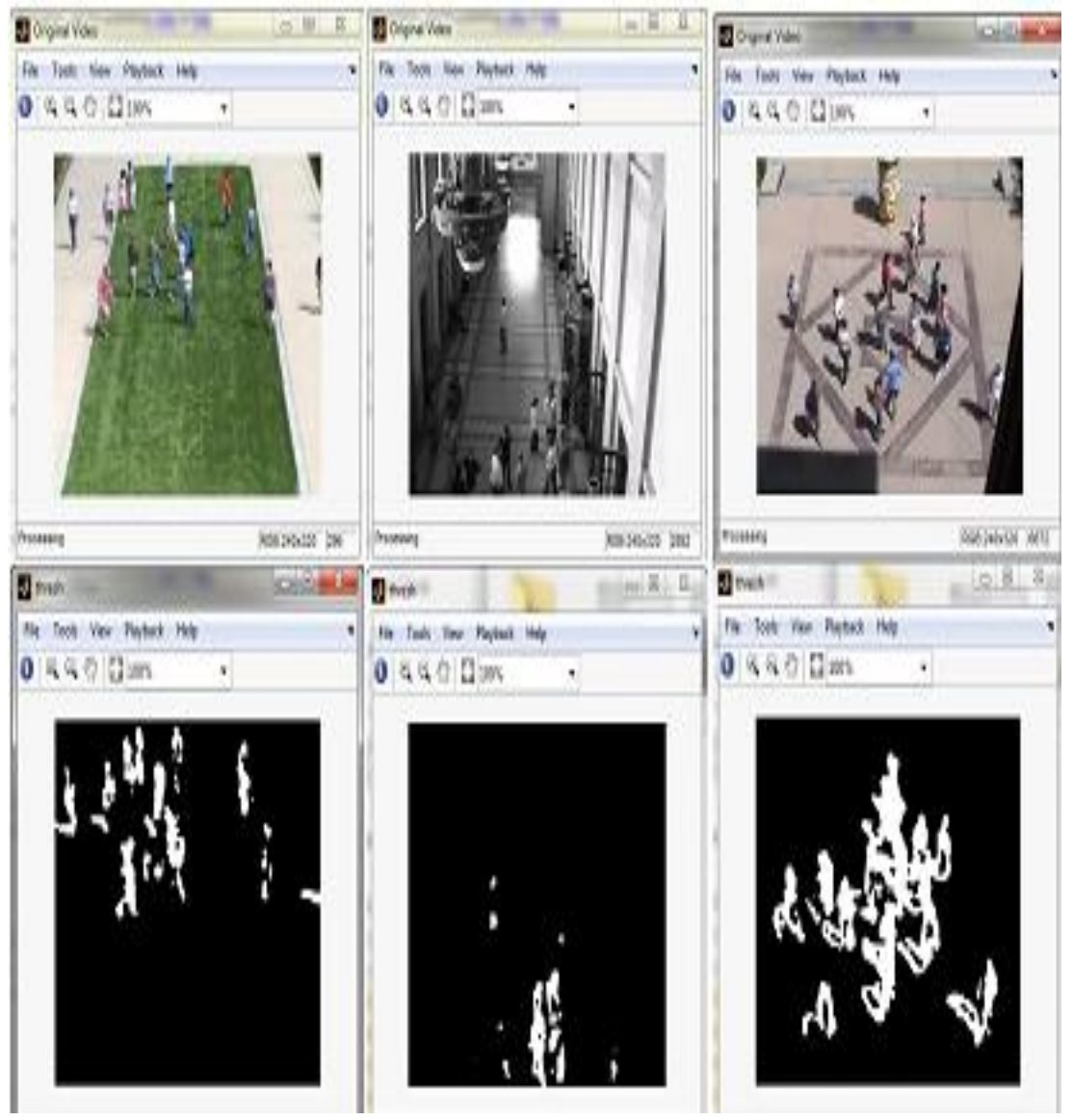

Figure 5

Figure 5 shows Background subtraction of the frames from the original video. The top right to left indicates the original video and the bottom right to left indicates the background separated frames.

The output result of the anomaly detection consists of the four blocks where the first blockconsists of the original input video followed by the pedestrian detection then the SCDimage andfinally the anomaly detection. The Figure 6 shows the output for the anomaly detection which is marked in red color.

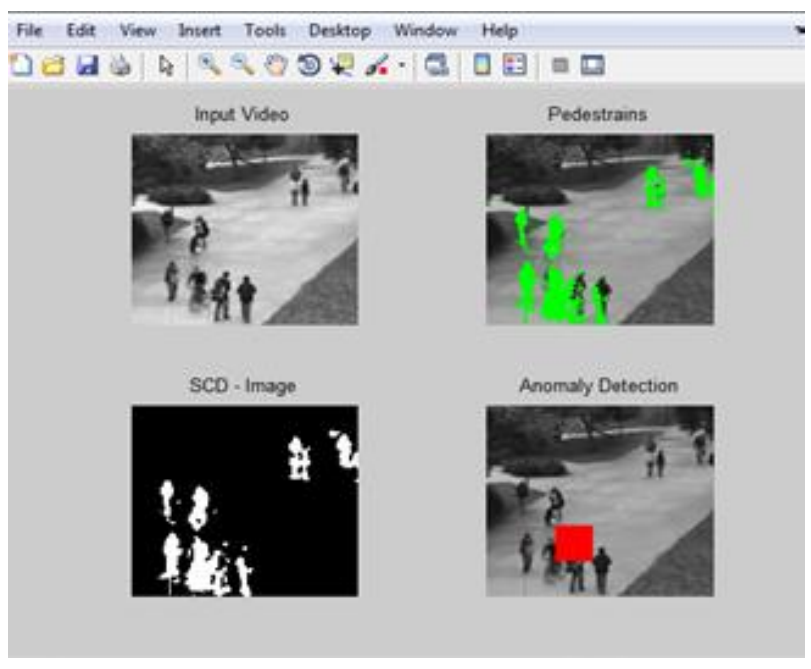

Figure 6: Anomaly Detection 


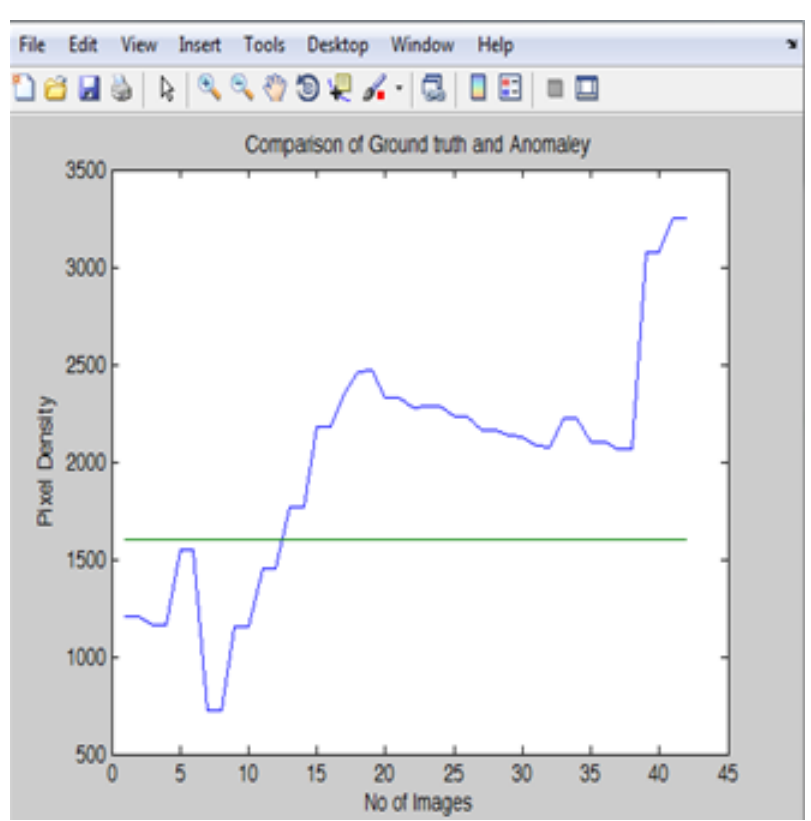

Figure 7: The Graphical Representation for Comparison of Ground Truth and Anomaly

The graph represented in the figure 7 explains the comparison of the ground truth and the anomaly. The $\mathrm{X}$-axis has number of images and the $\mathrm{Y}$-axis consists of the pixel density.

\section{CONCLUSION}

The abnormal behavior detection method from the crowd structure modelling is being proposed. The pedestrian detection highlights the every people present in the scenario. The SCD is being explored which discloses the individual in the crowded scene and depicts the relation amongst each of them. From the result of testing dataset, anomaly is detected.

\section{ACKNOWLEDGEMENT}

This work was supported by Government of Karnataka Vision Group on Science and Technology with project grant K-FIST (Level-I) (Project No. GRD-353) and Computer Vision Laboratory of Sahyadri College of Engineering \& Management, Mangalore.

\section{REFERENCES}

[1] Yuan Yuan, Jianwu Fang and Qi Wang, "Online Anomaly Detection in Crowd Scenes via Structure Analysis", IEEE Transactions on Cybernetics, Vol. 45, No. 3, March 2015.

[2] Teng Li, Huan Chang, Meng Wang, Bingbing Ni, Richang Hong and Shuicheng Yan, "Crowded Scene Analysis:A Survey", IEEE Transactions on Circuits and systems for video technology, Vol. 25, No. 3, March 2015.

[3] Dong-Gyu Lee, Heung-Il Suk, Sung-Kee Park and Seong-Whan Lee, , "Motion Influence Map for Unusual Human Activity Detection and Localization in Crowded Scenes", IEEE transactions on circuits and systems for video technology, October 2015

[4] B. Yogameena, K. Sindhu Priya, "Synoptic Video Based Human Crowd Behavior Analysis for Forensic Video Surveillance", Proc of IEEE Advances in Pattern Recognition (ICAPR), 2015.

[5] R. Mehran, A. Oyama and M. Shah, "Abnormal crowd behavior detection using social force model", Proc. IEEE Conf. Computer Vision. Pattern Recognition, Miami, FL, USA, Pp. 935-942, 2009.
[6] Shu Wang, Zhenjiang Miao and P.R. China, "Anomaly Detection in Crowd Scene”, Proc. of IEEE Signal Processing (ICSP), 2010.

[7] X. Cui, Q. Liu, M. Gao and D. Metaxas, "Abnormal detection using interaction energy potentials", Proc. IEEE Conf. Computer Vision. Pattern Recognition, Providence, RI, USA, Pp. 3161-3167, 2011.

[8] D.Y. Chen and P.C. Huang, "Visual-based human crowds behavior analysis based on graph modeling and matching", IEEE Sensors J., Vol. 13, No. 6, Pp. 2129-2138, Jun. 2013.

[9] S. Wu, B. Moore and M. Shah, "Chaotic invariants of lagrangian particle trajectories for anomaly detection in crowded scenes", Proc. IEEEConf. Comput. Vis. Pattern Recognit., San Francisco, CA, USA, Pp. 2054-2060, 2010.

[10] L. Kratz and K. Nishino, "Anomaly detection in extremely crowded scenes usingspatio-temporal motion pattern models", Proc. IEEE Conf. Comput. Vis. Pattern Recognit., Pp. 1446-1453, Jun. 2009.

[11] S. Ali and M. Shah, "A Lagrangian particle dynamics approach for crowd flow segmentation and stability analysis", Proc. IEEE Conf.Comput. Vis. Pattern Recognit., Pp. 1-6, Jun. 2007.

[12] L. Kratz and K. Nishino, "Anomaly detection in extremely crowded scenes usingspatio-temporal motion pattern models," in Proc. IEEE Conf. Comput. Vis. Pattern Recognit., Pp. 1446-1453, Jun. 2009.

[13] Y. Cong, J. Yuan and J. Liu, "Abnormal event detection in crowded scenes using sparse representation", Pattern Recognit., Vol. 46, No. 7, Pp. 1851-1864, 2013.

[14] M.J. Roshtkhari and M.D. Levine, "An on-line, real-time learning method for detecting anomalies in videos using spatio-temporal compositions", Comput. Vis. Image Understand., Vol. 117, No. 10, Pp. 1436-1452, 2013.

[15] Rajatsingh, SarveshVishwakarma, AnupamAgrawal, M. D. Tiwari, “ Unusual activity detection for video surveillance", IITM '10: Proceedings of the First International Conference on Intelligent Interactive Technologies and Multimedia, December 2010.

[16] Sarvesh Vishwakarma and Anupam Agrawal, "A survey on activity recognition and behavior understanding in video surveillance", Vis. Comput., Vol. 29, No. 10, Pp. 983-1009, October 2013.

[17] Sarvesh Vishwakarma and Anupam Agrawal, "Representing Feature Quantization Approach Using Spatial Temporal Relation for Action Recognition”, Perc. Mach. Intell., Vol. 7143, No. 10, Pp. 98-105, 2016.

[18] Sarvesh Vishwakarma, Akshay Sapre and Anupam Agrawal, "Action Recognition using Cuboids of Interest Points", Proc. IEEE Conf. ICSPCC, Pp. 1-6, 2011.

[19] Sarvesh Vishwakarma and Anupam Agrawal, "A Novel Approach for Feature Quantization using One-dimensional Histogram", Proc. IEEE Conf., Pp. 1-4, 2011.

[20] Sarvesh Vishwakarma and Anupam Agrawal, "Human Action Recognition using Spatial Temporal based Cuboids", Proc. IEEE Conf. ICIIP, Pp. 1-6, 2011.

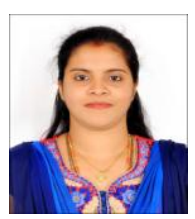

Pooja was born in Karnad on $8^{\text {th }}$ of August 1992. She perceived B.E. degree in Computer Science and engineering from Srinivas school of Engineering Mukka, Mangaluru at 2014. She is pursuing her MTech degree in Computer Science and Engineering from Sahyadri College of Engineering and Management Adyar, Mangaluru.

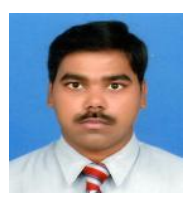

Sarvesh Vishwakarma received the B.Tech. degree in electronics and communication engineering from the University Institute of Engineering and Technology, Kanpur, India, in 2001, and the M.Tech. degree in computer science and engineering from the Indian Institute of Technology, Roorkee, India, in 2003. He obtained his $\mathrm{Ph} . \mathrm{D}$. degree in information technology with the Indian Institute of Information Technology, Allahabad, India. His research interests include computer vision, image processing, pattern recognition, and artificial intelligence applied to unusual activity analysis and surveillance applications. $\mathrm{He}$ is a member of the IEEE Computer Society. 